\title{
Depressive Mood and Fatigue in Iranian Patients With Spinal Cord Injury and Spared Walking Ability
}

\author{
Marzieh Matin ${ }^{1}$; Sahar Latifi ${ }^{1, *}$; Davood Koushki ${ }^{2}$; Abbas Norouzi Javidan ${ }^{1}$; Leila Laleh ${ }^{1}$; \\ Zahra Soltani ${ }^{1}$; Fereshteh Rahdari ${ }^{1}$ \\ ${ }^{1}$ Brain and Spinal Injury Research Center (BASIR), Tehran University of Medical Sciences, Tehran, IR Iran \\ ${ }^{2}$ Iran University of Medical Sciences, Tehran, IR Iran \\ ${ }^{*}$ Corresponding author: Sahar Latifi, Brain and Spinal Injury Research Center (BASIR), Imam Hospital, Keshavarz Boulevard, Gharib street, P. O. Box: 6114185, Tehran, IR Iran. Tel. +98- \\ 2166581504/+98-9125353259, Fax:+98-2166938885, E-mail: s-latifi@farabi.tums.ac.ir, saharlatifi_med@yahoo.com
}

Received: May 26, 2014; Revised: May 28, 2014; Accepted:13 Jul 2014

\begin{abstract}
Background: Fatigue and depression are common after spinal cord injury (SCI). However, their extent in individuals with spared ability to walk (assisted or nonassisted) has not yet been described in Iranian patients.

Objectives: Our purpose was to evaluate fatigue, depressive mood, and neuropathic pain among Iranian patients with spared ability. Patients and Methods: Patients with traumatic SCI who had spared walking ability with spinal cord independence scores (SCIM) higher than 65, referred to Brain and Spinal Injury Research Center (BASIR) between September 2012 and October 2013 were entered to the study. Fatigue, depressive mood and pain were evaluated using modified fatigue impairment scale (MFIS) for SCI (MFIS-SCI), Beck depression inventory (BDI), and 0-10 numerical rating scale(NRS), respectively.

Results: Among 30 patients who entered this investigation, $66.7 \%$ had normal moods (BDI $<10$ ) and only $3.3 \%$ showed severe depression (BDI:31-40). The mean total score of MFIS-SCI was 22.46 \pm 15.76 . Higher scores of BDI were significantly associated with higher scores of MFIS$\mathrm{SCI}$ in all cognitive $(\mathrm{P}<0.0001, \mathrm{r}=0.81)$, physical $(\mathrm{P}=0.001, \mathrm{r}=0.58)$ and psychosocial $(\mathrm{P}=0.001, \mathrm{r}=0.57)$ domains. Based on the previously measured cutoff point in MFIS-SCI, 53.3\% of our patients were fatigued. Patients with cervical level injury had significantly higher fatigue impacts (P: 0.036).

Conclusions: This study showed that a majority of patients with traumatic SCI who had spared ability of walking were fatigued, while depressive mood was less frequent among these patients. Neuropathic pain was not a determinant for fatigue and depression. Although the extent of physical activity was relatively similar among these patients, those with higher injury levels had higher fatigue impacts.
\end{abstract}

Keywords:Fatigue; Depression; Pain; Spinal Cord Injuries

\section{Background}

The association between fatigue and spinal cord injury (SCI) has been described (1-3), but poorly categorized. Some studies have detected more severe fatigue in tetraplegics (4) with complete lesions, while others have expressed the maximum fatigue in paraplegic individuals (5). Although many factors such as age and injury duration $(6,7)$ affect the fatigue severity in patients with SCI, still it seems that description of fatigue and its features is better to be defined within certain populations with their specific characteristics. In this field, classification of patients with SCI according to their ability to walk (with canes, walker or orthoses) and their spinal cord independence score (SCIM) lead to a proper categorization of these patients into subgroups based on their abilities. Previously, development of fatigue and depression were described in patients with SCI, regardless of their abilities (8). Here, we investigated only individuals with SCI with ability to walk (assisted or nonassisted) with high SCIM score, so that their restricted physical ability could be related to fatigue development and not to innerva- tion-induced immobility. To our knowledge, this was the first study describing fatigue in this specific population in Iran.

Besides fatigue (9), depressive mood and pain are also known causes of limited physical activity in patients with SCI (10). Depressive disorders are the most common psychological problems among these patients (11); even subclinical level of depression affects daily living activities (12). However, assessment of depressive disorders in individuals with SCI with ability to walk had not yet been performed in Iran and it was not clear whether these patients also had depressive mood and fatigue while their abilities to move and walk were spared at an acceptable level.

\section{Objectives}

Our purpose was to evaluate fatigue, depressive mood, and pain in Iranian individuals with spinal cord injury with high SCIM score and spared walking ability, using

Copyright (C) 2015, Tehran University of Medical Sciences. This is an open-access article distributed under the terms of the Creative Commons Attribution-NonCommercial 4.0 International License (http://creativecommons.org/licenses/by-nc/4.0/) which permits copy and redistribute the material just in noncommercial usages, provided the original work is properly cited. 
modified fatigue impairment scale (MFIS) for SCI (MFISSCI), Beck depression inventory, and 0-10 numerical rating scale (NRS), respectively.

\section{Patients and Methods}

\subsection{Study Design}

This was a cross-sectional study for people with chronic traumatic SCI. This investigation was based on obtaining information using standard questionnaires within faceto-face interviews. These interviews were held during patients' routine visits and participation was voluntary. The study was approved by the Research Ethics Committee of Tehran University of Medical Sciences. Data was collected during September 2012 to October 2013 in the Brain and Spinal Injury Research Center (BASIR). The study protocol was confirmed by the ethical guidelines of the 1975 Declaration of Helsinki.

\subsection{Participants}

Patients with traumatic SCI who had spared walking ability (whether nonassisted or assisted with canes, walker or orthoses), referred to BASIR from September 2012 to October 2013 were selected. Informed consents were obtained before the enrollment. The inclusion criteria were: acceptable ability to walk (assisted or nonassisted), post-injury duration longer than one year, traumatic SCI, SCIM score higher than 65. The exclusion criteria were: nontraumatic SCI, disability to stand or walk, SCIM score lower than 65, previous history of psychological disorders before the injury incidence including major depressive disorder, bipolar mood disorder, psychosis, schizophrenia, generalized anxiety disorders, obsessive compulsive disorder, and sleep problems. None of the patients and their family members stated the mentioned psychological disorders during their interviews with a psychiatrist. By considering the depressive phase which mostly occurs in the acute phase of SCI and may continue up to one year (13), we excluded patients with post-injury durations of less than one year to omit the bias effect of acute phase depression development.

\subsection{Measurements}

\subsubsection{Spinal Cord Independence Measure}

We used spinal cord independence measure III to evaluate the patients' independency and ability for conventional daily activities. This measure has especially been designed for individuals with SCI to assess their ability in performing routine tasks (14). Among the three published versions of SCIM, the third edition has been shown to have adequate reliability and validity for spinal cord injured patients $(15,16)$. This instrument consists of three subscales: self-care (0-20), mobility (0-40 scores), and respiration and sphincter management (0-40 scores), which comes to a maximum score of 100 . Higher scores illustrate more independency.

\subsubsection{Modified Fatigue Impairment Scale With Modifi- cation for Spinal Cord Independence}

The modified fatigue impact scale (MFIS) is a reliable tool in measuring fatigue, especially in multiple sclerosis (MS) (17). The Persian version of this questionnaire had an acceptable reliability and reproducibility (18). However, an MFIS with modifications for SCI has been introduced recently (19). MFIS-SCI is a 21-item questionnaire, including three main domains: cognition (11 items; score: 0-44), physical (seven items; score: 0-28) and psychosocial (three items; score: 0-12). The scores range from 0 to 84 and higher scores illustrate a higher impact of fatigue. Items are summarized in Table 1.

\subsubsection{Beck Depression Inventory}

Beck depression inventory consists of 21 items and the score ranges from 0 to 63 (20). Higher scores indicate more severe depressive moods. Interpretations of the scores are as follows: 1-10: normal, 11-16: mild mood disturbance, 17-20: borderline clinical depression, 21-30: moderate depressive mood, 31-40: severe depressive mood, over 40: extreme depression.

\subsubsection{Pain Determination}

Numerical rating scale (NRS), ranging from 0 to 10 , is the most common subjective assessment method for pain determination, in which patients give scores to their pain levels. Score 0 indicates no pain, 1-3 show mild pain (nagging, annoying and slightly interfering with daily living activities), 4-6 illustrates moderate pain (interfering significantly with daily life activities), and 7-10 are related to severe pain (disabling and being unable to perform daily life activities, requiring analgesics). Although this scale is the most commonly used technique for neuropathic pain determination in clinical practices, it only has modest accuracy (21).

\section{Results}

Thirty patients with spinal cord injuries with spared abilities to walk (assisted and nonassisted) were entered to this study. It is noticeable that the ratio of patients with SCI who had spared mobility ability was less than the wheelchair-dependent patients. Population of patients with SCI in our referral center, to which patients from all over the country are referred, mostly consists of individuals dependent to wheelchairs and only a minority of patients with SCI have spared ability to walk. Hence, although 30 patients may seem low number, it can present the population of individuals with SCI with walking ability. 
Matin M et al.

\begin{tabular}{|c|c|c|c|c|c|}
\hline \multirow[t]{2}{*}{ Domain and Item } & \multicolumn{5}{|c|}{ Score } \\
\hline & Never & Rarely & Sometimes & Often & Almost Always \\
\hline \multicolumn{6}{|l|}{ Cognitive } \\
\hline I have been less alert & 0 & 1 & 2 & 3 & 4 \\
\hline I have had difficulty paying attention for long periods of time & 0 & 1 & 2 & 3 & 4 \\
\hline I have been unable to think clearly & 0 & 1 & 2 & 3 & 4 \\
\hline I have been forgetful & 0 & 1 & 2 & 3 & 4 \\
\hline I have had difficulty paying attention for short periods of time & 0 & 1 & 2 & 3 & 4 \\
\hline I have had difficulty making decisions & 0 & 1 & 2 & 3 & 4 \\
\hline I have been less motivated to do anything that requires thinking & 0 & 1 & 2 & 3 & 4 \\
\hline I have had trouble finishing tasks that require thinking & 0 & 1 & 2 & 3 & 4 \\
\hline I have had difficulty organizing my thoughts when doing things & 0 & 1 & 2 & 3 & 4 \\
\hline My thinking has slowed down & 0 & 1 & 2 & 3 & 4 \\
\hline I have had trouble concentrating & 0 & 1 & 2 & 3 & 4 \\
\hline \multicolumn{6}{|l|}{ Physical } \\
\hline I have had to pace myself in my physical activities & 0 & 1 & 2 & 3 & 4 \\
\hline I have been less motivated to do anything that requires physical effort & 0 & 1 & 2 & 3 & 4 \\
\hline I have had trouble maintaining physical effort for long periods & 0 & 1 & 2 & 3 & 4 \\
\hline I have had trouble maintaining physical effort for short periods & 0 & 1 & 2 & 3 & 4 \\
\hline I have been physically uncomfortable & 0 & 1 & 2 & 3 & 4 \\
\hline I have been less able to complete tasks that require physical effort & 0 & 1 & 2 & 3 & 4 \\
\hline I have needed to rest more often or for longer periods & 0 & 1 & 2 & 3 & 4 \\
\hline \multicolumn{6}{|l|}{ Psychosocial } \\
\hline I have avoided/eliminated certain tasks, activities and lifestyles & 0 & 1 & 2 & 3 & 4 \\
\hline I have been less motivated to participate in social activities & 0 & 1 & 2 & 3 & 4 \\
\hline I have been limited in my ability to do things & 0 & 1 & 2 & 3 & 4 \\
\hline
\end{tabular}

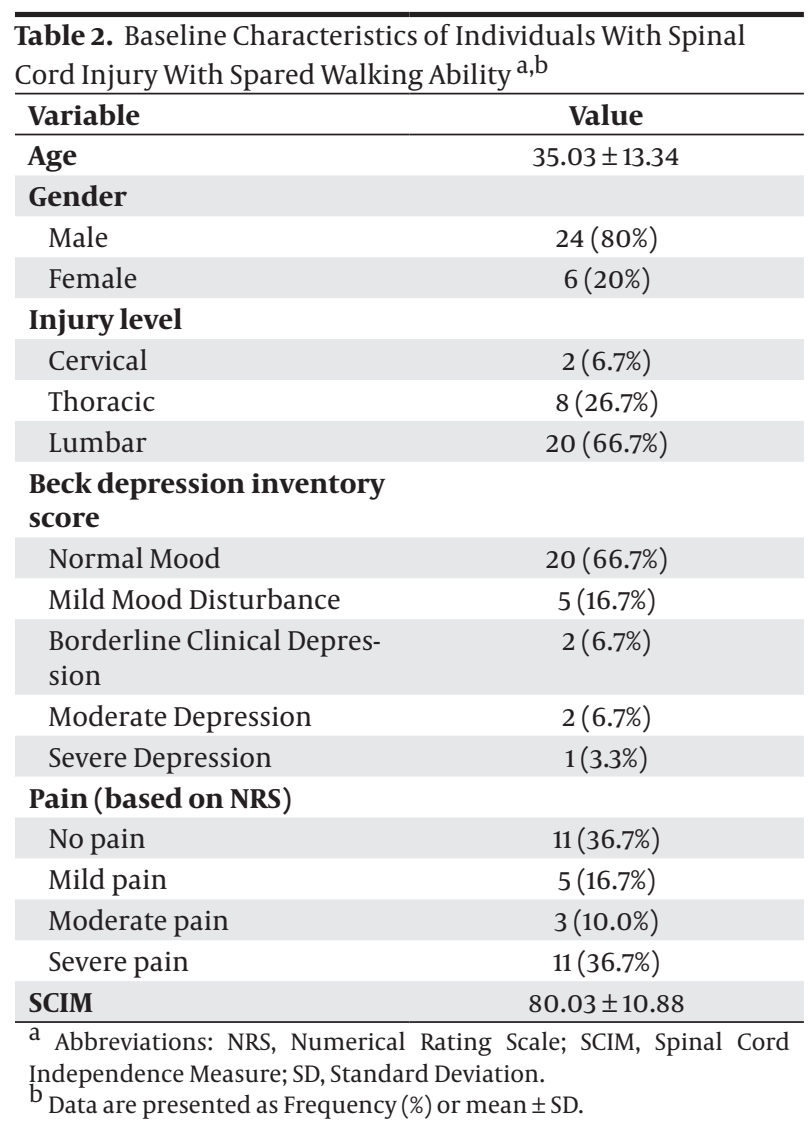

The examined population consisted of 24 (80\%) males and $6(20 \%)$ females. The mean age was $35.03 \pm 13.34$ (range: 18-60). The mean SCIM score was $80.03 \pm 10.88$ (range: 65-99). The mean BDI score was 9.46 \pm 9.05 (range: 0-38). Baseline characteristics are shown in Table 2.

Among patients with SCI with spared ability to walk, $66.7 \%(n=20)$ had BDI scores $<10$, considered as normal, 16.7\% $(n=5)$ had mild mood disturbances, 6.7\% $(n=2)$ showed borderline clinical depressive moods, $6.7 \%(\mathrm{n}=$ 2 ) revealed moderate depressive moods and only 1 (3.3\%) showed severe depression. No case of extreme depression (BDI score $>40$ ) was detected.

The obtained mean scores in cognitive, physical and psychosocial domains of MFIS-SCI were 6.87 \pm 6.96, $10.73 \pm$ 7.75 and $4.87 \pm 3.28$, respectively. The mean total score was $22.46 \pm 15.76$. Higher scores in BDI were significantly associated with higher scores of MFIS-SCI in all domains of cognitive $(\mathrm{P}<0.0001, \mathrm{r}=0.81)$, physical $(\mathrm{P}=0.001, \mathrm{r}=0.58)$ and psychosocial (P: 0.001, $\mathrm{r}=0.57$ ). This positive correlation between the results of BDI and the total MFIS-SCI score showed that patients with disturbed moods and higher depressive levels experienced higher fatigue impacts $(\mathrm{P}<$ $0.0001, r=0.76)$. The cutoff point to define fatigue patient by MFIS-SCI was 24.50 according to Imam et al. study (19). Based on this cutoff point, $53.3 \%$ of our patients $(n=16)$ were fatigued and $46.7 \%(n=14)$ did not reveal fatigue.

Although it is assumed that patients with higher levels of pain severity may experience higher fatigue impacts 
and depression, no significant association between the pain score and the BDI score was detected $(\mathrm{P}=0.63)$. The correlations between the pain score and the MFIS-SCI score in all three domains of cognitive, physical and psychosocial were statistically insignificant as well $(\mathrm{P}=0.42$, 0.34 and 0.39 , respectively). Age was not related to the BDI and MFIS-SCI scores ( $\mathrm{P}=0.60$ and 0.98 , respectively).

Injury level was another factor that showed a signifi- cant influence on the MFIS-SCI scores. It is well described that some patients with incomplete injury to spinal cord at cervical level may spare walking ability; but, still, it seems that patients with injury at cervical level experience more disability. In our study, patients with SCI at cervical level showed significantly higher MFIS scores in all the three domains, while no correlation was observed between the injury level and the BDI score (Table 3).

\begin{tabular}{|c|c|c|c|c|c|}
\hline \multirow[t]{2}{*}{ Variable } & \multicolumn{4}{|c|}{ MFIS-SCI } & \multirow[t]{2}{*}{ BDI } \\
\hline & Cognitive & Physical & Psychosocial & Total & \\
\hline \multicolumn{6}{|l|}{ Injury level } \\
\hline Cervical & $20.0 \pm 8.48$ & $17.50 \pm 3.53$ & $9.0 \pm 1.41$ & $46.50 \pm 13.43$ & $23.50 \pm 6.36$ \\
\hline Thoracic & $7.00 \pm 5.50$ & $9.38 \pm 8.94$ & $5.63 \pm 3.54$ & $24.0 \pm 16.21$ & $7.12 \pm 5.98$ \\
\hline Lumbar & $5.50 \pm 6.24$ & $9.80 \pm 7.45$ & $4.15 \pm 3.03$ & $19.45 \pm 14.19$ & $9.0 \pm 9.29$ \\
\hline Pvalue & 0.015 & 0.040 & 0.043 & 0.036 & 0.19 \\
\hline \multicolumn{6}{|l|}{ Pain } \\
\hline No pain & $5.55 \pm 7.00$ & $10.09 \pm 8.37$ & $4.55 \pm 3.47$ & $20.18 \pm 17.34$ & $8.90 \pm 10.80$ \\
\hline Mild pain & $7.60 \pm 4.61$ & $8.80 \pm 7.05$ & $4.40 \pm 4.09$ & $20.80 \pm 14.66$ & $7.60 \pm 7.95$ \\
\hline Moderate pain & $4.0 \pm 6.92$ & $4.00 \pm 3.606$ & $2.00 \pm 2.0$ & $10.00 \pm 9.53$ & $8.66 \pm 10.96$ \\
\hline Severe pain & $8.64 \pm 7.52$ & $14.09 \pm 7.30$ & $6.18 \pm 2.67$ & $28.90 \pm 14.7$ & $11.09 \pm 8.14$ \\
\hline P value & 0.38 & 0.26 & 0.30 & 0.25 & 0.56 \\
\hline
\end{tabular}

\section{Discussion}

Investigating fatigue is needed since it has a significant impact on daily function, social activities, and quality of life (22). Our study showed that the majority of patients with SCI with spared ability to walk were fatigued (53\%+). These results were comparable with Fawkes-Kirby's results (5), showing the prevalence of 57\% fatigue in individuals with SCI, regardless of their abilities. Here, we showed that the impact of fatigue among patients with SCI with spared ability to walk and high SCIM score did not significantly differ from that of total SCI population. Moreover, although it is commonly observed that patients with incomplete injuries have higher abilities, Fawkes-Kirby (5) showed that incomplete injuries lead to higher fatigue impacts. In consistence with our results, patients with SCI with higher SCIM scores and ability to walk did not show lower fatigue levels in comparison with the total SCI population's fatigue impact reported previously $(5,23)$. Although our investigation did not include the total SCI population for proper comparison, our results showed similarities with previously published studies (23-25). On the other hand, in contrast with the total SCI population, pain did not correlate significantly with fatigue impact. Fawkes-Kirby (5) showed that pain was one of the factors contributing to increasing fatigue, but our study showed no significant effect of pain on MFIS-SCI scores. Considering that the numerical assessment method of pain degree has only a modest accuracy (21), these outcomes should be cautiously interpreted.

Based on previous studies that revealed the mechanisms of muscle fatigue after SCI including compromised neuromuscular junction transmission (26) and substantial decrease in Na/K ATPase concentration (27), it is theoretically assumed that patients with more severe lesions and higher degrees of innervation should be more fatigued; but, we observed similarities of fatigue prevalence between patients with SCI with incomplete lesions and spared abilities to walk and other individuals with SCI. The reason is probably higher energy expenditure due to higher level of activity and increased musculoskeletal demand (28). In controversy with our results, Freixes et al. (29) showed only 19.2\% fatigue prevalence assessed by fatigue severity scale (FSS) in individuals with spinal cord injuries with American Spinal Injury Association (ASIA) impairment scale (AIS) D. However, Freixes's study showed a significant correlation between depression and fatigue, which was in line with our study. Same results supporting the positive correlation between fatigue and depression have been reported previously (30). Among the depressed non-SCI population, a cycle of low energy, fatigue, low mood, and reduced motivation to physical activity can be observed $(1,31)$. In individuals with spinal cord injuries, greater amounts of effort 
should be expended in daily activities. Hammell et al. (1) showed interconnections between high levels of fatigue (24), depression (32) and pain (33). Although these interconnections and associations have been previously shown by other investigations $(24,32)$, our results only revealed a positive correlation between fatigue and depression, while the effect of pain remained unnoticeable among patients with SCI with high levels of ability and independency. The reason of these controversies can be the use of subjective pain assessment with NRS, in which expression of pain severity by depressed or stressed individuals may be underestimated or exaggerated.

Depression is the most common psychological disorder in patients with SCI(11)and its occurrence after SCI is wellknown $(34,35)$. However, in our study population which only included patients with SCI with spared mobility and high SCIM score, $66.7 \%$ had normal moods assessed by BDI and only 3.3\% showed severe depressive moods. Up to now, there have been no available statistics about the prevalence of depression among Iranian patients with SCI; however, Sadeghirad et al. (36) reported the prevalence of major depressive disorder (MDD) in Tehran to be about $4.1 \%$ in a systematic review. As the prevalence of depression is related to many factors including social support and environmental circumstances, the specific relationship of disability condition with depressive episodes occurrence varies between different countries. In this regard, Kaviani et al. (37) assessed the depression prevalence in the general population of Tehran using Beck depression inventory and showed that $30.5 \%$ of females and $16.7 \%$ of inhabitant males in Tehran showed some degrees of mood disturbance. These results were comparable with our outcomes in patients with SCI with spared mobility, as 33\% of these patients revealed some levels of mood disturbance, assessed by BDI. It seems that the depression prevalence among these patients did not show a significant difference from the general population, and moreover, the obtained prevalence of severe depressive mood (3.3\%) was also similar to that of the general population of Tehran, reported by Sadeghirad et al. (4.1\%) (36). In conclusion, here, we reported that prevalence of depressive mood in Iranian patients with SCI with spared walking ability was similar to that of the general population of Tehran and it still has a significant correlation with the patients' experience of fatigue.

Our study showed that the injury level was significantly associated with the fatigue impact, as patients with injuries at cervical level revealed higher MFIS-SCI scores. Previously, Tawashy et al. (10) reported that injury level was not a determinant of physical activity, while Dearwater et al. (38) illustrated that paraplegics were more active than tetraplegics. In our study, although the ranges of activities among patients were relatively similar (as all patients were able to walk), patients with higher injury levels showed higher fatigue impacts. Fawkes-Kirby (5) suggested that higher level of fatigue appeared when the injury was more incomplete due to higher physical activity and energy ex- penditure; but here, we showed that even with relatively similar physical activity levels among patients with SCI, patients with higher injury levels were more fatigued.

Our study showed that a majority of patients with SCI who had incomplete lesions and spared abilities to walk were fatigued, based on the MFIS-SCI scores (53\%). Only $3.3 \%$ of these patients showed severe depressive moods, assessed by BDI. Depression was significantly correlated with fatigue impact, but the relationships between pain severity and scores of BDI and MFI-SCI were not significant. Although all the patients had relatively similar extents of physical activities and were able to walk (assisted or nonassisted), patients with injuries at cervical levels showed significantly higher fatigue impacts.

\subsection{Study Limitation}

This study did not compare the results of patients with SCI with spared walking abilities with other patients with SCI, and the interpretations were mostly performed by comparing these results with previously published outcomes in individuals with spinal cord injuries. The modified fatigue impairment scale (MFIS) has been validated in Farsi (18), but the MFIS modified for spinal cord injury (MFIS-SCI) has not yet been validated in Farsi. We recommend further investigations to validate the Farsi version of this questionnaire.

\section{Acknowledgements}

We would like to thank all the staff who helped us in the process of data collection.

\section{Authors' contributions}

Marzieh Matin contributed to the study design and data collection. Sahar Latifi contributed to performing the statistical analysis and writing the manuscript. Davood Koushki contributed to analyzing and editing the paper. Abbas Norouzi Javidan contributed to obtaining the funding. Leila Laleh, Zahra Soltani and Fereshteh Rahdari contributed to data collection.

\section{Funding/support}

This study was supported by Tehran University of Medical Sciences.

\section{References}

1. Hammell KW, Miller WC, Forwell SJ, Forman BE, Jacobsen BA. Fatigue and spinal cord injury: a qualitative analysis. Spinal Cord. 2009;47(1):44-9.

2. Pelletier CA, Hicks AL. Muscle fatigue characteristics in paralyzed muscle after spinal cord injury. Spinal Cord. 2011;49(1):125-30.

3. Barat M, Dehail P, de Seze M. Fatigue after spinal cord injury. Ann Readapt Med Phys. 2006;49(6):277-82.

4. Thompson L. Functional changes in persons aging with spinal cord injury. Assist Technol.1999;11(2):123-9.

5. Fawkes-Kirby TM, Wheeler MA, Anton HA, Miller WC, Townson AF, Weeks CA. Clinical correlates of fatigue in spinal cord injury. Spinal Cord. 2008;46(1):21-5. 
6. Charlifue SW, Gerhart KA, Whiteneck GG. Conceptualizing and quantifying functional change: an examination of aging with spinal cord injury. Top Geriatr Rehabil. 1998;13(3):35-48.

7. Pentland W, Walker J, Minnes P, Tremblay M, Brouwer B, Gould M. Women with spinal cord injury and the impact of aging. Spinal Cord. 2002;40(8):374-87.

8. Shields RK, Chang YJ, Dudley-Javoroski S, Lin CH. Predictive model of muscle fatigue after spinal cord injury in humans. Muscle Nerve. 2006;34(1):84-91.

9. Rosqvist E, Heikkinen E, Lyyra TM, Hirvensalo M, Kallinen M, Leinonen $\mathrm{R}$, et al. Factors affecting the increased risk of physical inactivity among older people with depressive symptoms. Scand J Med Sci Sports. 2009;19(3):398-405.

10. Tawashy AE, Eng JJ, Lin KH, Tang PF, Hung C. Physical activity is related to lower levels of pain, fatigue and depression in individuals with spinal-cord injury: a correlational study. Spinal Cord. 2009;47(4):301-6.

11. Elliott TR, Frank RG. Depression following spinal cord injury. Arch Phys Med Rehabil.1996;77(8):816-23.

12. Judd LL, Paulus MP, Wells KB, Rapaport MH. Socioeconomic burden of subsyndromal depressive symptoms and major depression in a sample of the general population. Am J Psychiatry. 1996;153(11):1411-7.

13. Ullrich PM, Lincoln RK, Tackett MJ, Miskevics S, Smith BM, Weaver FM. Pain, depression, and health care utilization over time after spinal cord injury. Rehabil Psychol. 2013;58(2):158-65.

14. Catz A, Itzkovich M, Steinberg F, Philo O, Ring H, Ronen J, et al The Catz-Itzkovich SCIM: a revised version of the Spinal Cord Independence Measure. Disabil Rehabil. 2001;23(6):263-8.

15. Anderson K, Aito S, Atkins M, Biering-Sorensen F, Charlifue S, Curt A, et al. Functional recovery measures for spinal cord injury: an evidence-based review for clinical practice and research. J Spinal Cord Med. 2008;31(2):133-44.

16. Catz A, Itzkovich M, Tesio L, Biering-Sorensen F, Weeks C, Laramee MT, et al. A multicenter international study on the Spinal Cord Independence Measure, version III: Rasch psychometric validation. Spinal Cord. 2007;45(4):275-91.

17. Mills RJ, Young CA, Pallant JF, Tennant A. Rasch analysis of the Modified Fatigue Impact Scale (MFIS) in multiple sclerosis. J Neurol Neurosurg Psychiatry. 2010;81(9):1049-51.

18. Ghajarzadeh M, Jalilian R, Eskandari G, Ali Sahraian M, Reza Azimi A. Validity and reliability of Persian version of Modified Fatigue Impact Scale (MFIS) questionnaire in Iranian patients with multiple sclerosis. Disabil Rehabil. 2013;35(18):1509-12.

19. Imam B, Anton HA, Miller WC. Measurement properties of a telephone version of the Modified Fatigue Impact Scale among individuals with a traumatic spinal cord injury. Spinal Cord. 2012;50(12):920-4.

20. Siegert RJ, Tennant A, Turner-Stokes L. Rasch analysis of the Beck Depression Inventory-II in a neurological rehabilitation sample. Disabil Rehabil. 2010;32(1):8-17.

21. Krebs EE, Carey TS, Weinberger M. Accuracy of the pain numeric rating scale as a screening test in primary care. J Gen Intern Med. 2007;22(10):1453-8.

22. Ingles JL, Eskes GA, Phillips SJ. Fatigue after stroke. Arch Phys Med Rehabil. 1999;80(2):173-8.

23. Lee AK, Miller WC, Townson AF, Anton HA, F. N. Research Group . Medication use is associated with fatigue in a sample of community-living individuals who have a spinal cord injury: a chart review. Spinal Cord. 2010;48(5):429-33.

24. Jensen MP, Kuehn CM, Amtmann D, Cardenas DD. Symptom burden in persons with spinal cord injury. Arch Phys Med Rehabil. 2007;88(5):638-45.

25. Suzuki R, Krahn GL, McCarthy MJ, Adams EJ. Understanding health outcomes: Physical secondary conditions in people with spinal cord injury. Rehabil Psychol. 2007;52(3):338.

26. Ollivier-Lanvin K, Lemay MA, Tessler A, Burns AS. Neuromuscular transmission failure and muscle fatigue in ankle muscles of the adult rat after spinal cord injury. J Appl Physiol (1985). 2009;107(4):1190-4.

27. Ditor DS, Hamilton S, Tarnopolsky MA, Green HJ, Craven BC, Parise G, et al. Na+,K+-ATPase concentration and fiber type distribution after spinal cord injury. Muscle Nerve. 2004; 29(1):38-45.

28. Kemp B, Thompson L. Aging and spinal cord injury: medical, functional, and psychosocial changes. SCI Nurs. 2002;19(2):51-60.

29. Freixes O, Rivas ME, Agrati PE, Bochkezanian V, Waldman SV, Olmos LE. Fatigue level in spinal cord injury AIS D community ambulatory subjects. Spinal Cord. 2012;50(6):422-5.

30. Widerstrom-Noga EG, Felipe-Cuervo E, Yezierski RP. Chronic pain after spinal injury: interference with sleep and daily activities. Arch Phys Med Rehabil. 2001;82(11):1571-7.

31. Chris Williams. Overcoming Depression and Low Mood: A Five Areas Approach.London: Hodder Arnold; 2006.

32. Kemp BJ, Adkins RH, Thompson L. Aging with a spinal cord injury: what recent research shows. Top Spinal Cord Inj Rehabil . 2004;10(2):175-97.

33. Henwood P, Ellis JA. Chronic neuropathic pain in spinal cord injury: the patient's perspective. Pain Res Manag. 2004;9(1):39-45.

34. Bombardier CH. Depression and spinal cord injury. Arch Phys Med Rehabil. 2014;95(2):413-4.

35. Hartoonian N, Hoffman JM, Kalpakjian CZ, Taylor HB, Krause $\mathrm{JK}$, Bombardier $\mathrm{CH}$. Evaluating a spinal cord injury-specific model of depression and quality of life. Arch Phys Med Rehabil. 2014;95(3):455-65.

36. Sadeghirad B, Haghdoost AA, Amin-Esmaeili M, Ananloo ES, Ghaeli P, Rahimi-Movaghar A, et al. Epidemiology of major depressive disorder in iran: a systematic review and meta-analysis. Int J Prev Med. 2010;1(2):81-91.

37. Kaviani H, Nazari H, Hormozi K. Prevalence Of Depressive Disordrs In Tehran Resident Population (year 2000). Tehran Univ Med J. 2002;60(5):393-9.

38. Dearwater SR, LaPorte RE, Cauley JA, Brenes G. Assessment of physical activity in inactive populations. Med Sci Sports Exerc. 1985;17(6):651-5. 